\title{
BMJ Open Multicentre observational study of adults with asthma exacerbations: who are the frequent users of the emergency department in Japan?
}

\author{
Hiroko Watase, ${ }^{1}$ Yusuke Hagiwara, ${ }^{1,2}$ Takuyo Chiba, ${ }^{1,3}$ Carlos A Camargo Jr, ${ }^{4}$ \\ Kohei Hasegawa, ${ }^{1,4}$ on behalf of the Japanese Emergency Medicine Network \\ Investigators
}

To cite: Watase $\mathrm{H}$,

Hagiwara Y, Chiba T, et al. Multicentre observational study of adults with asthma exacerbations: who are the frequent users of the emergency department in Japan? BMJ Open 2015;5: e007435. doi:10.1136/ bmjopen-2014-007435

- Prepublication history for this paper is available online. To view these files please visit the journal online (http://dx.doi.org/10.1136/ bmjopen-2014-007435).

Received 11 December 2014 Revised 18 March 2015 Accepted 23 March 2015

CrossMark

For numbered affiliations see end of article.

Correspondence to Dr Hiroko Watase; hiroko7@uw.edu

\section{ABSTRACT}

Objectives: Emergency department (ED) visits for asthma exacerbation reflect a failure of longitudinal asthma management. However, little is known about the characteristics of patients with frequent ED visits ( $\geq 2$ visits in a 1 -year period). We aimed to characterise the adult patients who frequently presented to the ED for asthma exacerbation in Japan.

Design: A multicentre chart review study of 23 EDs across Japan.

Participants: Adults aged 18-54 years who presented to the ED with asthma exacerbation from 2009 to 2011.

Outcome measures: Frequency of ED visits for asthma exacerbation in a 1-year period, including the index ED visit.

Results: Of the 1002 eligible patients, $218(22 \%)$ had frequent $E D$ visits, accounting for $48 \%$ of total $E D$ visits for asthma exacerbation in the 1-year period.

Specifically, $12 \%$ had 2 ED visits and $10 \%$ had $\geq 3$ visits. In these patients, guideline-recommended chronic management was suboptimal. For example, among patients with $\geq 3 \mathrm{ED}$ visits, only $63 \%$ were treated with inhaled corticosteroids and $49 \%$ were current smokers. In a multinomial logistic regression model, markers of chronic asthma severity (history of hospitalisation for asthma and use of inhaled corticosteroids) were significantly associated with a higher frequency of $E D$ visits (both $p<0.05$ ).

Conclusions: This multicentre study in Japan demonstrated that many patients are frequent ED users for asthma exacerbation. We also found that their asthma control management is suboptimal, most likely contributing to worse chronic severity and more frequent $E D$ visits. Further dissemination and adoption of evidence-based guidelines are required to reduce asthma morbidity in this high-risk population.

\section{INTRODUCTION}

Asthma affects an estimated 300 million people worldwide, with 250000 annual deaths attributed to this disease. ${ }^{1}$ Recent estimates indicated

\section{Strengths and limitations of this study}

- This is a large multicentre study from 23 emergency departments (EDs) across different geographical regions of Japan.

- This is the first study to investigate the characteristics of patients with frequent ED visits for asthma exacerbation in Japan.

- Our study population mainly consisted of urban, academic EDs in Japan. Therefore, our findings may not be generalisable to rural, non-academic EDs or other healthcare settings.

that approximately $4 \%$ ( 5 million) of Japanese adults receive a diagnosis of asthma in their lifetime, ${ }^{2}$ contributing to substantial health and economic burdens. ${ }^{3}{ }^{4}$ Furthermore, asthma exacerbations account for a significant proportion of this burden, resulting in estimated annual costs of $\$ 3.4$ billion in Japan. ${ }^{4}$ In this context, the Japanese government set a national goal to prevent asthma exacerbations and asthma-related mortality. ${ }^{5}$

As most of the asthma exacerbations are deemed to be preventable with high-quality prevention-oriented outpatient care, frequent (2 or more in a 1-year period) emergency department (ED) visits reflect a failure of asthma control management. ${ }^{6}$ Our previous studies in the USA investigated this highrisk population, along with risk factors of frequent ED visits for asthma exacerbations, such as non-white race/ethnicity, low socioeconomic status and higher chronic asthma severity. ${ }^{6-10}$ However, little is known about the characteristics of patients with frequent ED visits for asthma exacerbation in other industrialised nations, particularly in those with universal healthcare systems.

To address this knowledge gap in the literature, using data from a 23-centre observational 
study of adults presenting to the ED with asthma exacerbation, we aimed to characterise the patients with frequent ED visits for asthma exacerbation in Japan.

\section{METHODS}

\section{Study design and settings}

We conducted a multicentre chart review study to characterise the clinical epidemiology of asthma exacerbations in Japanese EDs. The study setting, methods and variables have been reported previously. ${ }^{11}$ Briefly, this study was coordinated by the Japanese Emergency Medicine Network (JEMNet, http://jemnet.asia/wp) in collaboration with the US-based Emergency Medicine Network (http://www.emnet-usa.org). JEMNet is a consortium of 23 academic and community medical centres from different geographic regions across Japan. The participating institutions had a median annual ED census of 29000 patient visits (range $12000-67000$ ) and 107 visits with asthma exacerbation (range 20-200). All 23 EDs were staffed by ED-based attending physicians. The Institutional Review Board of each participating centre and Massachusetts General Hospital approved this study with a waiver of written informed consent.

\section{Selection of participants}

Using the International Classification of Diseases, Tenth Revision, Clinical Modification (ICD-10-CM) code J45.xx, ${ }^{12}$ the study sites identified all ED visits with a principal ED or hospital discharge diagnosis of asthma from hospital administrative records from January 2009 until December 2011 (figure 1). The inclusion criteria of this study were asthma ED visits made by patients aged 18-54 years with a history of physician diagnosis of asthma prior to the index ED visit. We excluded ED visits made by patients with a history of physician diagnosis of chronic obstructive pulmonary disease, emphysema, or chronic bronchitis, transfer ED visits, ED visits not primarily prompted by an asthma exacerbation, or repeat ED visits by the same individual. In the case of repeat visits, only the first ED visit during the study period was included. Therefore, each ED visit in the study represented a unique patient in the ED. These criteria were the same as in our previous US-based studies on this topic. ${ }^{6} 8$

Preceding 365 days

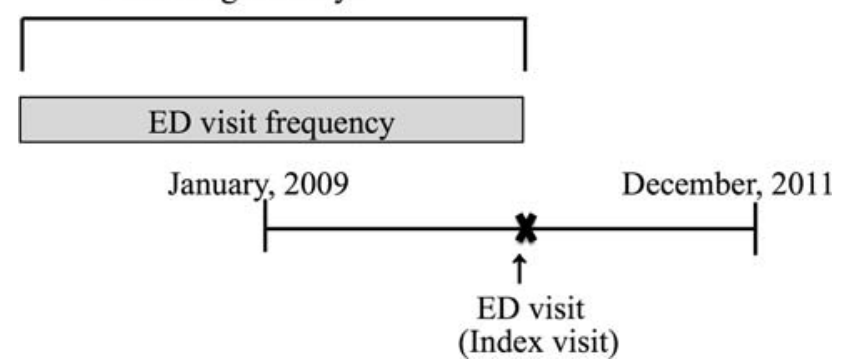

Figure 1 The schematic of strategy used to identify emergency department (ED) visit frequency.

\section{Data collection}

Using a standardised form, ${ }^{6} 1113$ on-site chart abstractors reviewed $60 \mathrm{ED}$ charts that are randomly selected from the eligible patients identified by using the ICD-10-CM code. We collected information on patient demographics, chronic asthma history and chronic asthma medications, as well as details of the current asthma exacerbation, ED management and ED disposition. ${ }^{11}$

Sites with less than 60 charts during this period also reviewed eligible charts from the calendar years 2009 and 2010 to reach the target number of 60 charts. All chart abstractors were emergency physicians or emergency medicine residents. Abstractors were trained with a $1 \mathrm{~h}$ lecture, and then they completed practice charts, which were assessed versus a criterion standard. If an abstractor's accuracy was less than $80 \%$ per chart, the individual was retrained. All forms were reviewed by site investigators and submitted to a secure, web-based, electronic database. Then these data were reviewed at the JEMNet Coordinating Center, and site investigators were queried about missing data and discrepancies identified by manual data checks.

\section{Outcome measures}

The outcome measure of interest was the frequency of ED visits for asthma exacerbation in a 1-year period (ie, the number of ED visits for asthma exacerbation during the preceding 365 days including the index visit; figure 1).

\section{Statistical analysis}

For the purpose of this analysis, we classified patients into three ED-using groups based on the distribution of ED visit frequency and the literature: ${ }^{10}$ one ED visit (ie, index ED visit only), two ED visits and three or more ED visits for asthma exacerbation in the 1-year period. First, we examined unadjusted associations between the patient-level characteristics and the outcomes by using a $\chi^{2}$ test, a one-way analysis of variance and a Kruskal-Wallis test, where appropriate. Second, to examine independent predictors of a higher frequency of ED visits for asthma exacerbation, we constructed a multinomial logistic regression model, with one ED visit as the reference for each. The patient-level factors included in the model were age, sex, smoking, chronic asthma factors (history of hospital admission and intubation for asthma exacerbation) and chronic asthma medications (inhaled corticosteroids (ICS), oral xanthines and leukotriene modifiers).

Additionally, we performed a sensitivity analysis to assess the robustness of the association between patientlevel factors and the frequency of ED visits. We fit a negative binomial regression model to account for statistical overdispersion and to avoid the use of an arbitrary cut-off point for the frequency of ED visits for asthma exacerbation. Incidence rate ratios (IRRs) are presented with $95 \%$ CIs. Two-sided $\mathrm{p}<0.05$ was considered statistically significant. All analyses were performed using Stata 13.0 software (StataCorp; College Station, Texas, USA). 


\section{RESULTS}

We created a cohort of 1380 patients who presented to 1 of the 23 EDs with an asthma exacerbation during 2009-2011; 91\% of the ED visits were made in 2011. Among these patients, 1002 (73\%) had data on the frequency of ED visits for asthma exacerbation in the previous year, and were included in this analysis. The analytic and non-analytic cohorts were similar in age, sex, ED presentation and ED disposition (all $\mathrm{p}>0.05$ : data not shown).

\section{Frequent ED visits for asthma exacerbation}

Of the 1002 patients in the analytic cohort, 784 (78\%; 95\% CI $76 \%$ to $81 \%$ ) reported one ED visit, while 117 (12\%; $95 \%$ CI $10 \%$ to $14 \%$ ) had two ED visits and 101 (10\%; $95 \%$ CI $8 \%$ to $12 \%$ ) had three or more ED visits in the 1-year period. The number of patients with asthma exacerbation and the cumulative number of ED visits for asthma exacerbation in the 1-year period by ED visit frequency are shown in figure 2. Patients with two ED visits accounted for $16 \%$ (95\% CI $14 \%$ to $18 \%$ ) of all ED visits for asthma exacerbation in the 1-year period, and patients with three or more ED visits accounted for $32 \%$ (95\% CI $29 \%$ to $34 \%$ ).

\section{Patient characteristics by the ED visit frequency group}

Chronic asthma factors, chronic asthma medications and ED management differed among the ED visit frequency groups (table 1). Patients with three or more ED visits for asthma exacerbation were more likely to be a current smoker; indeed, approximately half of them were current smokers. Likewise, patients with frequent

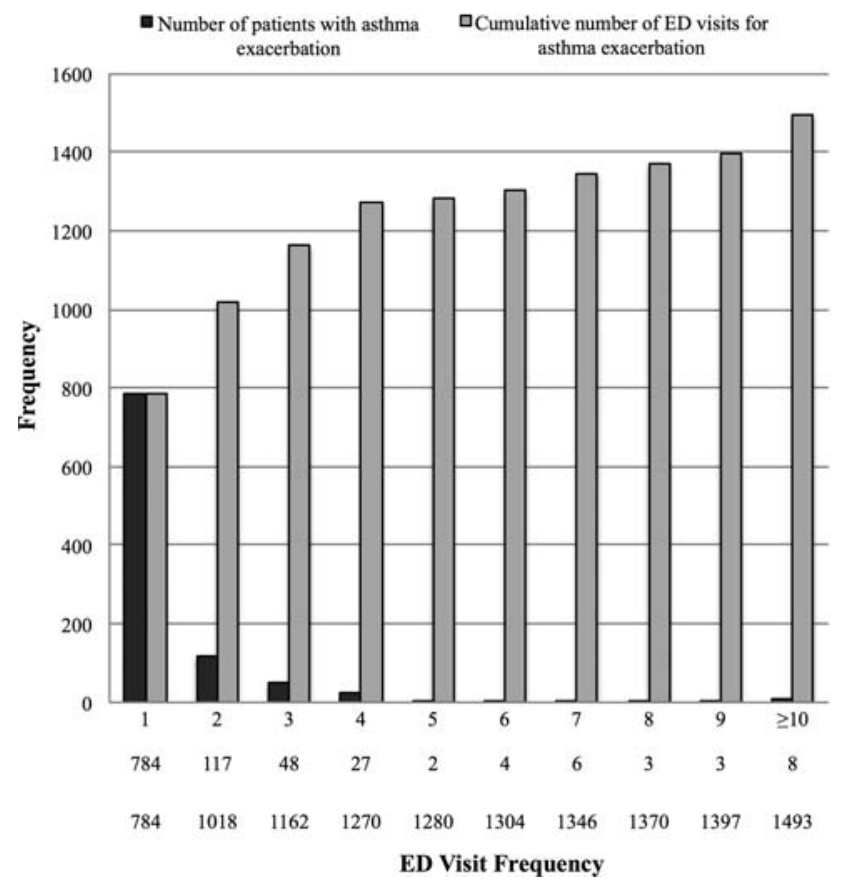

Figure 2 Number of patients with asthma exacerbation and cumulative number of emergency department (ED) visits for asthma exacerbation, according to ED visit frequency.
ED visits for asthma exacerbation were more likely to have a history of oral corticosteroid use, hospital admission and intubation for asthma, and hospital admission for asthma in the past year (all $\mathrm{p}<0.001$ ). Similarly, these patients were more likely to have been on ICS, oral xanthines and leukotriene modifiers (all $\mathrm{p}<0.001$ ). However, the proportion of patients who had used these long-term control medications was relatively low among the frequency groups. For example, only $45 \%$ of the patients with two ED visits and $63 \%$ of the patients with three or more ED visits were currently treated with ICS.

Although there were no significant between-group differences in the markers of acute severity (eg, respiratory rates and peak flow measurement at the ED presentation), patients with a higher frequency of $\mathrm{ED}$ visits were more likely to be treated with systemic corticosteroids during their index ED visit $(\mathrm{p}<0.001)$. In contrast, there was no significant difference in ED disposition among the three groups $(\mathrm{p}=0.62)$.

\section{Factors associated with frequent ED visits for asthma exacerbation}

In the multinomial logistic regression model, history of hospital admission for asthma and current use of ICS remained significant as independent predictors of a higher frequency of ED visits across the frequency groups (table 2). Likewise, with the use of a negative binomial regression model (table 3), history of hospital admission for asthma (IRR 3.07; 95\% CI 2.07 to 4.56; $\mathrm{p}<0.001$ ), current use of ICS (IRR 2.06; 95\% CI 1.36 to 3.14; $\mathrm{p}=0.001$ ) and current use of oral xanthines (IRR 1.72; $95 \%$ CI 1.02 to $2.91 ; \mathrm{p}=0.04$ ) were independently associated with a higher frequency of ED visits for asthma exacerbation.

\section{DISCUSSION}

This multicentre study is the first to characterise patients with frequent ED visits for asthma exacerbation in Japan. Among 1002 patients presenting with asthma exacerbation to 1 of the 23 Japanese EDs, we found that $22 \%$ had frequent ED visits, accounting for $48 \%$ of total ED visits for asthma exacerbation in the 1-year period. We also found that the frequent ED users received suboptimal asthma control management. Indeed, more than one-third of patients with three and more ED visits were not treated with ICS and half were current smokers, most likely contributing to worse chronic severity and more frequent ED visits for asthma exacerbation.

\section{Results in context}

Previous studies in the USA reported many patients with frequent ED visits for asthma exacerbation. In the 1990s, $73-82 \%$ of patients in the ED with asthma exacerbation had frequent ED visits in the previous year, ${ }^{78}$ while the number was $46 \%$ in the 2010 s. ${ }^{6}$ In contrast, our study in Japan demonstrated that $22 \%$ of patients in the ED with asthma exacerbation had frequent ED visits in the 1-year 
Table 1 Patient demographics, chronic asthma factors, acute asthma presentation and emergency department (ED) course for asthma exacerbation, according to the number of ED visits in the 1-year period

\begin{tabular}{|c|c|c|c|c|}
\hline Variables* & $\begin{array}{l}1 \text { ED visit } \\
(n=784)\end{array}$ & $\begin{array}{l}2 \text { ED visits } \\
(n=117)\end{array}$ & $\begin{array}{l}\geq 3 \text { ED visits } \\
(n=101)\end{array}$ & p Value \\
\hline Age (years), median (IQR) & $34(26-43)$ & $34(25-42)$ & $37(27-43)$ & 0.45 \\
\hline Male sex & $43(40-47)$ & $41(32-50)$ & $39(29-49)$ & 0.64 \\
\hline Current smoker & $42(39-46)$ & $32(24-42)$ & $49(38-59)$ & 0.045 \\
\hline \multicolumn{5}{|l|}{ Chronic asthma factors } \\
\hline History of oral corticosteroid use for asthma & $35(31-40)$ & $75(64-84)$ & $84(75-91)$ & $<0.001$ \\
\hline History of hospital admission for asthma & $23(20-27)$ & $50(39-61)$ & $66(55-75)$ & $<0.001$ \\
\hline History of intubation for asthma & $1(1-3)$ & $1(0-7)$ & $8(3-16)$ & $<0.001$ \\
\hline History of hospital admission for asthma in the past year & $2(1-3)$ & $19(12-28)$ & $34(25-45)$ & $<0.001$ \\
\hline Current use of inhaled corticosteroids & $23(20-26)$ & $45(36-55)$ & $63(53-72)$ & $<0.001$ \\
\hline Current use of oral xanthines & $10(8-12)$ & $12(7-20)$ & $26(18-36)$ & $<0.001$ \\
\hline Current use of leukotriene modifiers & $8(6-10)$ & $16(10-25)$ & $29(21-39)$ & $<0.001$ \\
\hline \multicolumn{5}{|l|}{ Acute asthma presentation } \\
\hline \multicolumn{5}{|l|}{ Duration of symptoms } \\
\hline$<4 \mathrm{~h}$ before ED arrival & $16(14-19)$ & $13(7-20)$ & $21(13-31)$ & 0.08 \\
\hline $4-23 \mathrm{~h}$ & $43(40-47)$ & $49(39-58)$ & $53(42-63)$ & \\
\hline $1-7$ days & $34(31-38)$ & $36(27-46)$ & $22(14-32)$ & \\
\hline$>7$ days & $7(5-9)$ & $3(1-8)$ & $4(1-10)$ & \\
\hline \multicolumn{5}{|l|}{ ED course } \\
\hline Initial respiratory rate (breath/min), mean (SD) & $22 \pm 6$ & $21 \pm 6$ & $24 \pm 6$ & 0.07 \\
\hline Initial oxygen saturation (\%), mean (SD) & $95 \pm 3$ & $95 \pm 4$ & $95 \pm 4$ & 0.12 \\
\hline Initial peak flow† (L/min), mean (SD) & $214 \pm 103$ & $254 \pm 63$ & $170 \pm 57$ & 0.21 \\
\hline Number of inhaled $\beta$-agonists in first hour, median (IQR) & $1(1-2)$ & $1(1-2)$ & $1(1-2)$ & 0.33 \\
\hline Number of inhaled $\beta$-agonists over ED stay, median (IQR) & $1(1-2)$ & $1(1-2)$ & $2(1-2)$ & 0.17 \\
\hline Received systemic corticosteroid treatment & $41(37-44)$ & $44(34-53)$ & $62(52-72)$ & $<0.001$ \\
\hline ED disposition & & & & 0.62 \\
\hline Sent home & $89(86-91)$ & $86(79-92)$ & $89(81-94)$ & \\
\hline Hospital admission & $11(9-13)$ & $12(7-19)$ & $11(6-19)$ & \\
\hline Other (eg, left against medical advice) & $1(0-1)$ & $2(0-6)$ & 0 & \\
\hline Sent home with systemic corticosteroids & $35(32-39)$ & $29(20-39)$ & $32(23-43)$ & 0.42 \\
\hline
\end{tabular}

period. The reasons for this apparent difference between the US and Japanese studies are most likely multifactorial. Potential explanations include differences in study design, setting and population, although our recent multicentre observational study of US adults for
2011-2012 used the same design as the current study. ${ }^{6}$ Differences in healthcare systems may also partly explain the differences. The Japanese healthcare system provides universal healthcare. ${ }^{1415}$ Additionally, most primary care offices in Japan do not require an appointment to see

Table 2 Multinomial models of factors associated with frequent emergency department (ED) visits for asthma exacerbation

\begin{tabular}{|c|c|c|c|c|}
\hline \multirow[b]{2}{*}{ Variables } & \multicolumn{2}{|l|}{2 (vs 1) ED visits } & \multicolumn{2}{|l|}{$\geq 3$ (vs 1 ) ED visits } \\
\hline & Risk ratio $(95 \% \mathrm{Cl})$ & p Value & Risk ratio (95\% Cl) & p Value \\
\hline \multicolumn{5}{|l|}{ Age, years } \\
\hline $18-29$ & 1 (reference) & & 1 (reference) & \\
\hline 30-39 & 0.76 (0.39 to 1.48$)$ & 0.43 & 0.81 (0.41 to 1.60$)$ & 0.55 \\
\hline $40-54$ & 0.73 (0.39 to 1.39$)$ & 0.34 & $0.86(0.46$ to 1.61$)$ & 0.64 \\
\hline Male sex & 0.67 (0.38 to 1.19$)$ & 0.17 & 0.74 (0.43 to 1.29$)$ & 0.29 \\
\hline Current smoker & 1.91 (1.01 to 3.58$)$ & 0.04 & 1.86 (0.97 to 3.57$)$ & 0.06 \\
\hline History of hospital admission for asthma & 3.06 (1.72 to 5.45$)$ & $<0.001$ & $4.77(2.73$ to 8.32$)$ & $<0.001$ \\
\hline History of intubation for asthma & $0.36(0.04$ to 3.15$)$ & 0.36 & 1.19 (0.37 to 3.84$)$ & 0.29 \\
\hline Current use of inhaled corticosteroids & $2.03(1.10$ to 3.74$)$ & 0.02 & $2.10(1.17$ to 3.79$)$ & 0.01 \\
\hline Current use of oral xanthines & 0.79 (0.30 to 2.06$)$ & 0.63 & 1.75 (0.85 to 3.56$)$ & 0.13 \\
\hline Current use of leukotriene modifiers & $1.39(0.57$ to 3.37$)$ & 0.47 & 2.63 (1.31 to 5.28$)$ & 0.01 \\
\hline
\end{tabular}


Table 3 Negative binomial regression models of factors associated with frequent emergency department visits for asthma exacerbation

\begin{tabular}{llc}
\hline Variables & Incident rate ratio (95\% Cl) & p Value \\
\hline Age, years & & \\
18-29 & 1 (reference) & 0.42 \\
$30-39$ & $0.83(0.52$ to 1.31$)$ & 0.60 \\
$40-54$ & $0.89(0.58$ to 1.37$)$ & 0.19 \\
Male sex & $0.78(0.53$ to 1.13$)$ & 0.42 \\
Current smoker & $1.19(0.78$ to 1.82$)$ & $<0.001$ \\
History of hospital admission for asthma & $\mathbf{3 . 0 7}(\mathbf{2 . 0 7}$ to 4.56$)$ & 0.40 \\
History of intubation for asthma & $1.51(0.58$ to 3.93$)$ & $\mathbf{0 . 0 0 1}$ \\
Current use of inhaled corticosteroids & $\mathbf{2 . 0 6 ( 1 . 3 6 \text { to } 3 . 1 4 )}$ & $\mathbf{0 . 0 4}$ \\
Current use of oral xanthines & $\mathbf{1 . 7 2 ( 1 . 0 2 \text { to } 2 . 9 1 )}$ & 0.05 \\
Current use of leukotriene modifiers & $1.73(0.99$ to 3.00$)$ &
\end{tabular}

physicians, ${ }^{15}$ and primary care offices frequently serve as urgent care clinics. Accordingly, Japanese patients with asthma may have chosen to visit their primary care office instead of the ED when they had asthma exacerbations, thereby reducing the number of frequent ED visits in this study.

\section{Suboptimal longitudinal management in frequent ED users} In agreement with the previous studies, ${ }^{6-9}$ we found that patients with frequent ED visits for asthma exacerbation were more likely to have markers of severe chronic asthma-for example, history of hospital admission for asthma, and current use of ICS. It is clinically plausible that patients with more severe asthma have more frequent ED visits for asthma exacerbation. ${ }^{16}$ However, we were struck by the finding that long-term asthma treatment in this high-risk population was suboptimal. Indeed, our data demonstrated that only $45 \%$ of the patients with two ED visits and $63 \%$ of the patients with three or more ED visits for asthma exacerbation were treated with ICS. This observation was consistent with the literature demonstrating that the ICS use in patients with asthma remains low in Japan compared with that in other developed nations, although its rate has been increasing (12\% in 2001 to $18 \%$ in 2005). ${ }^{17}$

We also found that approximately half of the patients with three or more ED visits for asthma exacerbation in the 1-year period were current smokers. It is well documented that cigarette smoke exposure is a risk factor for asthma exacerbation. ${ }^{18-21}$ Accordingly, international and national guidelines for asthma recommend smoking cessation and avoiding smoke exposure. ${ }^{22-24}$ Our findings collectively suggest that only a subset of these high-risk patients in Japan received optimal long-term asthma control treatment and education, most likely contributing to worse chronic asthma severity and more frequent ED visits for asthma exacerbation. Therefore, further dissemination and adoption of evidence-based guidelines are required to reduce the large burden of asthma morbidity in Japan.

\section{Potential limitations}

Our study has several potential limitations. First, our study population mainly consisted of urban, academic EDs in Japan. Therefore, our findings may not be representative of patients with asthma in rural, non-academic EDs or generalisable to the other developed nations. Second, $27 \%$ of patients were excluded owing to a lack of information on the frequency of ED visits for asthma exacerbation in the previous year. However, the analytic and non-analytic cohorts were similar in the patient demographics, ED presentation and ED disposition. These similarities argue against a significant selection bias. Third, this is a chart review study; therefore, the quality of information could be deficient owing to misclassification. However, a prior ED-based study reported high agreement in the patient characteristics between chart review and directly observed care in the patients presenting to the ED for asthma exacerbation, with the א-statistics from 0.50 to $0.82 .{ }^{25}$ Furthermore, in this multicentre study, the inter-rater agreement was moderate to perfect ( -statistics, 0.56-1.00). ${ }^{11}$ Fourth, as with any observational studies, the observed associations with frequent ED visits do not necessarily prove causality and might be explained, at least partly, by unmeasured confounders, such as the amount of inhaled $\beta$-agonist or ICS use before the ED visit and time-variant covariates (eg, ICS use over time). Finally, as described above, patients with asthma exacerbation might have presented to their primary care office rather than the ED, thereby leading to an underestimation of the burden of patients with frequent asthma exacerbations. However, since our focus was on the burden of the frequent ED utilisation, our findings are of direct relevance to optimise asthma management in this high-risk population.

\section{CONCLUSIONS}

In this 23-centre observational study of ED adult patients with asthma exacerbation in Japan, we found that many patients had two or more ED visits for asthma exacerbation, accounting for approximately half of the total ED 
visits for asthma exacerbation in the 1-year period. Our data also demonstrated that asthma control management in this high-risk population is suboptimal, most likely contributing to worse chronic severity and more frequent ED visits for asthma exacerbation. For researchers, our observations should facilitate further investigation into the quality of the longitudinal asthma control care and research on barriers to the delivery of evidence-based asthma care in Japan. For clinicians and professional organisations, continuous efforts to promote dissemination and adoption of evidence-based guidelines-for example, development of a nationwide asthma programme focusing on early intervention and disease control $^{26} 27$-are required to reduce asthma morbidity in this high-risk population.

\section{Author affiliations}

${ }^{1}$ Department of Radiology, University of Washington, Seattle, Washington, USA

2Department of Emergency Medicine, Tokyo Metropolitan Children's Medical Center, Tokyo, Japan

${ }^{3}$ Department of Emergency Medicine, University of Massachusetts Medical School, Worcester, Massachusetts, USA

${ }^{4}$ Department of Emergency Medicine, Massachusetts General Hospital, Harvard Medical School, Boston, Massachusetts, USA

Acknowledgements We thank the participating investigators for their ongoing dedication to emergency medicine research.

JEMNet study members The site principal investigators were: Yukikazu Awaya, MD (Yahata Municipal Hospital, Kitakyushu, Fukuoka); Tadahiro Goto, MD (Fukui University Hospital, Eiheiji, Fukui); Nao Hanaki, MD (Japanese Redcross Nagoya Second Hospital, Nagoya, Aichi); Takuro Hayashi, MD (Kobe City Medical Center General Hospital, Kobe, Hyogo); Taichi Imamura, MD (Shonan Kamakura General Hospital); Yusuke Kodama, MD (Kyoritsu General Hospital, Nagoya, Aichi); Takahiro Kondo, MD (Ekisaikai General Hospital, Nagoya, Aichi); Yasuhiro Koyama, MD (St. Marianna University Hospital, Kawasaki, Kawasaki); Tomomichi Matsuda, MD (Sapporo East Tokushukai Hospital, Sappro, Hokkaido); Nobuhiro Miyamae, MD (Rakuwakai Otowa Hospital, Kyoto, Kyoto); Miki Morikawa, MD (Juntendo University Urayasu Hospital, Urayasu, Chiba); Hideya Nagai, MD (Fukui Prefectural Hospital, Fukui, Fukui); Masahumi Ohta, MD (Kensei Hospital, Tsugaru, Aomori); Hiroshi Okamoto, MD (Kurashiki Central Hospital, Kurashiki, Okayama); Yuichi Okano, MD (Japanese Redcross Kumamoto Hospital, Kumamoto, Kumamoto); Masashi Okubo, MD (Okinawa Prefectural Chubu Hospital, Uruma, Okinawa); Yutaka Saito, MD (Kawasaki City Hospital Kawasaki, Kawasaki); Hiroyuki Suzuki, MD (Fukuoka Tokushukai Hospital, Kasuga, Fukuoka); Sei Takahashi, MD (Kinikyo Chuo Hospital, Sappro, Hokkaido); Kotaro Takebe, MD (Kyoto Prefectural University Hospital, Kyoto, Kyoto); Kenzo Tanaka, MD, MPH (Kameda Medical Center, Kamogawa, Chiba); Yuki Sato, MD (Nigata City General Hospital, Nigata, Nigata); and Hideto Yasuda, MD (Japanese Redcross Musashino Hospital, Musashino, Tokyo).

Contributors HW contributed in the study concept and design, analysis and interpretation of the data, and drafting of the manuscript. YH and TC contributed in the study concept and design, acquisition of the data, and critical revision of the manuscript for important intellectual content. CAC contributed in the study concept and design, analysis and interpretation of the data, and critical revision of the manuscript for important intellectual content. $\mathrm{KH}$ contributed in the study concept and design, interpretation of the data, and critical revision of the manuscript for important intellectual content.

Funding This study was supported by a grant from St. Luke's Life Science Institute (Tokyo, Japan).

Competing interests CAC has provided asthma-related consultation for GlaxoSmithKline, Merck, Novartis and Teva.

Provenance and peer review Not commissioned; externally peer reviewed.
Data sharing statement No additional data are available.

Open Access This is an Open Access article distributed in accordance with the Creative Commons Attribution Non Commercial (CC BY-NC 4.0) license, which permits others to distribute, remix, adapt, build upon this work noncommercially, and license their derivative works on different terms, provided the original work is properly cited and the use is non-commercial. See: http:// creativecommons.org/licenses/by-nc/4.0/

\section{REFERENCES}

1. Centers for Disease Control and Prevention. Asthma. http://www. cdc.gov/asthma/ (accessed 10 Mar 2015).

2. Fukutomi $\mathrm{Y}$, Nakamura $\mathrm{H}$, Kobayashi $\mathrm{F}$, et al. Nationwide cross-sectional population-based study on the prevalences of asthma and asthma symptoms among Japanese adults. Int Arch Allergy Immunol 2010;153:280-7.

3. Weiss KB, Sullivan SD, Lyttle CS. Trends in the cost of illness for asthma in the United States, 1985-1994. J Allergy Clin Immunol 2000;106:493-9.

4. Adachi M, Fukuda T, Morikawa A, et al. [Asthma mortality and burden of asthma in Japan]. Allergology \& Immunology 2005; 12:1438-47.

5. Ministry of Health, Labor and Welfare. http://www.mhlw.go.jp/ new-info/kobetu/kenkou/ryumachi/jititai.html (accessed 10 Mar 2015).

6. Hasegawa K, Sullivan AF, Hirashima ET, et al. A multicenter observational study of US adults with acute asthma: who are the frequent users of the emergency department? J Allergy Clin Immunol Pract 2014:2:733-40.

7. Ford JG, Meyer IH, Sternfels P, et al. Patterns and predictors of asthma-related emergency department use in Harlem. Chest 2001;120:1129-35.

8. Griswold SK, Nordstrom CR, Clark S, et al. Asthma exacerbations in North American adults: who are the "frequent fliers" in the emergency department? Chest 2005;127:1579-86.

9. Pines JM, Buford K. Predictors of frequent emergency department utilization in Southeastern Pennsylvania. J Asthma 2006;43:219-23.

10. Hasegawa K, Tsugawa $\mathrm{Y}$, Brown DF, et al. A population-based study of adults who frequently visit the emergency department for acute asthma. California and Florida, 2009-2010. Ann Am Thorac Soc 2014:11:158-66.

11. Hasegawa K, Chiba T, Hagiwara $\mathrm{Y}$, et al. Quality of care for acute asthma in emergency departments in Japan: a multicenter observational study. J Allergy Clin Immunol Pract 2013;1:509-15. e1-3.

12. National Center for Health Statistics. International Classification of Diseases, Tenth Revision, Clinical Modification (ICD-10-CM). http:// www.cdc.gov/nchs/icd/icd10cm.htm (accessed 10 Mar 2015).

13. Hasegawa K, Sullivan AF, Tsugawa $\mathrm{Y}$, et al. Comparison of US emergency department acute asthma care quality: 1997-2001 and 2001-2012. J Allergy Clin Immunol 2014;135:73-80.

14. OECD. Health at a Glance 2013. http://www.oecd-ilibrary.org/ social-issues-migration-health/health-at-a-glance-2013_health_ glance-2013-en (accessed 10 Mar 2015).

15. Ikegami N, Campbell JC. Japan's health care system: containing costs and attempting reform. Health Aff (Millwood) 2004:23:26-36.

16. Chipps BE, Zeiger RS, Borish L, et al. Key findings and clinical implications from The Epidemiology and Natural History of Asthma: Outcomes and Treatment Regimens (TENOR) study. J Allergy Clin Immunol 2012;130:332-42.e10.

17. Adachi M, Ohta K, Morikawa A, et al. [Changes in asthma insights and reality in Japan (AIRJ) in 2005 since 2000]. Arerugi 2008;57:107-20.

18. Osborne ML, Pedula KL, O'Hollaren $\mathrm{M}$, et al. Assessing future need for acute care in adult asthmatics: the Profile of Asthma Risk Study: a prospective health maintenance organization-based study. Chest 2007:132:1151-61.

19. Silverman RA, Boudreaux ED, Woodruff PG, et al. Cigarette smoking among asthmatic adults presenting to 64 emergency departments. Chest 2003;123:1472-9.

20. Siroux V, Pin I, Oryszczyn MP, et al. Relationships of active smoking to asthma and asthma severity in the EGEA study. Epidemiological study on the genetics and environment of asthma. Eur Respir $J$ 2000;15:470-7.

21. Marquette $\mathrm{CH}$, Saulnier $\mathrm{F}$, Leroy $\mathrm{O}$, et al. Long-term prognosis of near-fatal asthma. A 6-year follow-up study of 145 asthmatic patients 
who underwent mechanical ventilation for a near-fatal attack of asthma. Am Rev Respir Dis 1992;146:76-81.

22. Global Initiative for Asthma (GINA). http://www.ginasthma.org/ (accessed 10 Mar 2015)

23. National Asthma Education and Prevention Program. Expert Panel Report 3 (EPR-3): guidelines for the diagnosis and management of asthma-summary report 2007. J Allergy Clin Immunol 2007;120: S94-138.

24. Ohta $\mathrm{K}$, Ichinose $\mathrm{M}$, Nagase $\mathrm{H}$, et al. Japanese guideline for adult asthma 2014. Allergol Int 2014;63:293-333.
25. McDermott MF, Lenhardt RO, Catrambone CD, et al. Adequacy of medical chart review to characterize emergency care for asthma: findings from the Illinois Emergency Department Asthma Collaborative. Acad Emerg Med 2006;13:345-8.

26. Haahtela T, Valovirta E, Kauppi P, et al. The Finnish Allergy Programme 2008-2018-scientific rationale and practical implementation. Asia Pacific Allergy 2012;2:275-9.

27. Kauppi P, Linna M, Martikainen J, et al. Follow-up of the Finnish Asthma Programme 2000-2010: reduction of hospital burden needs risk group rethinking. Thorax 2013;68:292-3. 